01

\title{
Метод диагностики обобщенной синхронизации в системах со сложной топологией хаотического аттрактора
}

\author{
() О.И. Москаленко, В.А. Ханадеев, А.А. Короновский
}

Саратовский национальный исследовательский государственный университет им. Н.Г. Чернышевского, Саратов, Россия

E-mail: o.i.moskalenko@gmail.com

\section{Поступило в Редакцию 17 мая 2018 г.}

Предложен метод диагностики режима обобщенной синхронизации в системах со сложной топологией хаотического аттрактора, основанный на рассмотрении трубок траекторий в фазовом пространстве взаимодействующих систем. Работоспособность метода проверена путем численного моделирования двух взаимно связанных модифицированных систем Лоренца, одна из которых находится в хаотическом режиме, а другая демонстрирует режим гиперхаоса. Результаты сопоставлены с данными, полученными методом расчета спектра показателей Ляпунова. Получено хорошее согласие между ними.

DOI: 10.21883/PJTF.2018.19.46687.17391

Одним из наиболее распространенных типов хаотической синхронизации в природе и технике является режим обобщенной хаотической синхронизации [1-3]. Этот тип синхронного поведения означает наличие функциональной связи (функционала) между состояниями взаимодействующих осцилляторов и может наблюдаться как в однонаправленно, так и во взаимно связанных системах и сетях [1-5], при этом возможно его использование для скрытой передачи информации, в том числе по каналам связи с высоким уровнем шума [6-8].

Для диагностики режима обобщенной хаотической синхронизации предложено несколько методов и подходов: метод вспомогательной системы [9], метод расчета спектра показателей Ляпунова [10], метод ближайших соседей [1] и др. Метод вспомогательной системы справедлив только для однонаправленно связанных систем (он не может быть применен в случае систем с взаимным типом связи [11]) и основан на введении в рассмотрение идентичной копии одной из 
взаимодействующих систем (ведомой системы), что затрудняет его использование в эксперименте. В то же время разработанная в [12] модификация этого подхода позволяет диагностировать обобщенную синхронизацию в данном случае путем подачи одного и того же предварительно записанного сигнала на ведомую систему или путем введения линии задержки [8]. Для взаимно связанных систем, как отмечалось выше, этот метод неприменим, однако два других указанных подхода (расчет показателей Ляпунова и метод ближайших соседей) работают как в случае однонаправленной, так и в случае взаимной связи $[4,11,13]$. Диагностирование обобщенной синхронизации при помощи расчета спектра показателей Ляпунова возможно по моменту перехода как минимум второго по старшинству показателя Ляпунова в область отрицательных значений $[4,10]$, что затрудняет применение данного подхода к экспериментальным временны́ рядам в связи со сложностями расчета показателей Ляпунова, отличных от старшего, по временно́му ряду из-за накопления ошибки численного счета [14].

Метод ближайших соседей является самым универсальным. Он может быть применен к любым системам независимо от типа связи между ними и даже предназначен для анализа экспериментальных временны́х рядов $[1,4]$. Согласно данному подходу, необходимо зафиксировать несколько опорных состояний в фазовом пространстве одной из взаимодействующих систем, найти их ближайших соседей и посмотреть, каким образом будут вести себя их образы в фазовом пространстве другой системы. Если для всех опорных состояний образы ближайших соседей будут локализованы в ограниченных областях аттрактора, можно говорить о наличии обобщенной синхронизации в системе. Если такая закономерность наблюдается только для части опорных точек, в системе диагностируется режим перемежающейся обобщенной синхронизации. Если образы ближайших соседей оказываются разбросанными случайным образом по всему аттрактору, в системе диагностируется асинхронный режим.

Одним из принципиальных недостатков метода ближайших соседей является невозможность точного определения порогового значения установления синхронного режима. Метод позволяет установить лишь факт наличия/отсутствия режима обобщенной синхронизации, а следовательно, используется, как правило, для подтверждения работоспособности других методов и подходов, например при расчете спектра показателей Ляпунова для систем с взаимным типом связи [4,13]. 


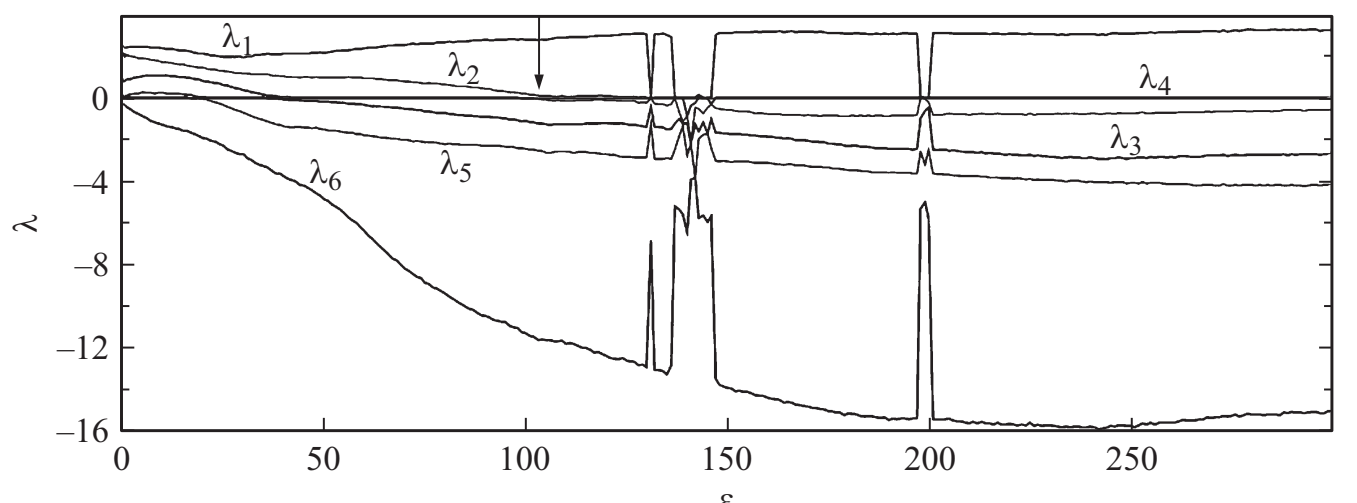

Рис. 1. Зависимости шести старших показателей Ляпунова для системы (1) от параметра связи $\varepsilon$. Значение параметра связи, соответствующее установлению режима обобщенной синхронизации, показано стрелкой. 
Другим недостатком этого метода, как показано далее, является невозможность его применения к системам с достаточно сложной топологией аттрактора, например аттракторам с двулистной структурой (как, например, у аттрактора Лоренца, системы Чуа и др. [15-18]): из-за перескока изображающей точки с одного листа хаотического аттрактора на другой метод ближайших соседей не позволяет диагностировать режим обобщенной синхронизации даже в том случае, когда этот режим уже установился.

В настоящей работе для диагностики режима обобщенной синхронизации в системах со сложной топологией хаотического аттрактора (в том числе по экспериментальным временны́ рядам) предложено использовать метод фазовых трубок [3], суть которого заключается в рассмотрении трубок траекторий в фазовом пространстве взаимодействующих систем. Метод по своей сути является модификацией метода ближайших соседей, за тем лишь исключением, что при поиске ближайших соседей учитываются не все ближайшие состояния системы, а только те, которые остаются близкими на всей длине „предыстории“ (длине фазовой трубки), длительность которой определяется абсолютной величиной показателя Ляпунова, отвечающего за установление режима обобщенной синхронизации. Покажем работоспособность данного подхода на примере системы двух взаимно связанных модифицированных систем Лоренца [18]:

$$
\left\{\begin{array}{l}
\dot{x}_{1,2}=a\left(y_{1,2}-x_{1,2}\right)+e y_{1,2} z_{1,2}, \\
\dot{y}_{1,2}=c x_{1,2}-d x_{1,2} z_{1,2}+y_{1,2}+u_{1,2} \\
\dot{z}_{1,2}=x_{1,2} y_{1,2}-b z_{1,2} \\
\dot{u}_{1,2}=-k_{1,2} y_{1,2}+\varepsilon\left(x_{2,1}-x_{1,2}\right)
\end{array}\right.
$$

где $a=35, b=4.9, c=25, d=5, e=35, k_{1}=110, k_{2}=190-$ управляющие параметры, $\varepsilon$ - параметр связи. На рис. 1 представлена зависимость шести старших показателей Ляпунова от параметра связи. Видно, что при увеличении силы связи в системе происходит последовательный переход двух положительных показателей Ляпунова в область отрицательных значений. Когда оба показателя Ляпунова становятся отрицательными, т.е. при $\varepsilon>105$, в системе диагностируется режим обобщенной синхронизации. Чтобы доказать наличие обобщенной синхронизации в данном случае воспользуемся методом ближайших соседей. На рис. 2 представлены фазовые портреты взаимодействующих

Письма в ЖТФ, 2018, том 44, вып. 19 
систем при значении параметра связи $\varepsilon=250$, что соответствует режиму достаточно „сильной“ обобщенной синхронизации. На фазовом портрете первой системы (рис. 2,a) показаны три случайные точки, выбранные в качестве опорных, и их ближайшие соседи. Очевидно, что они сосредоточены в ограниченных областях аттрактора, представляющих собой малые окрестности опорных точек. Аналогичная ситуация в соответствии с идеологией метода ближайших соседей должна наблюдаться и во второй системе, однако, как отмечалось выше, из-за сложной топологии хаотических аттракторов взаимодействующих систем образы ближайших соседей в фазовом пространстве второй системы оказываются разбросанными достаточно сильно по аттрактору (рис. $2, b$ ), что свидетельствует о неприменимости метода ближайших соседей в данном случае.

Метод фазовых трубок позволяет ликвидировать данную проблему. На рис. 3 приведены результаты применения метода фазовых трубок к исследуемой системе. Так же как и на рис. 2, здесь приведены фазовые портреты первой и второй модифицированных систем Лоренца (при тех же самых значениях управляющих параметров), те же опорные состояния, выбранные в фазовом пространстве первой системы, их ближайшие соседи (рис. 3,a) и их образы в фазовом пространстве второй системы (рис. 3, $b$ ). Длина фазовой трубки выбрана равной $\tau=0.03$. Видно, что даже при малой длине фазовой трубки образы ближайших соседей оказываются локализованными в ограниченных областях аттрактора, а размеры этих областей сопоставимы с размерами областей, занимаемых ближайшими соседями в фазовом пространстве первой системы. Полученные результаты свидетельствуют о применимости метода фазовых трубок для диагностики режима обобщенной синхронизации в системах со сложной топологией хаотических аттракторов. Эти результаты сопоставлены с данными, полученными методом расчета спектра показателей Ляпунова. Показано, что оба метода диагностируют наличие обобщенной синхронизации в данном случае.

Таким образом, в работе впервые показана возможность применения метода фазовых трубок для диагностики режима обобщенной синхронизации в системах со сложной топологией хаотического аттрактора, в то время как все остальные методы имеют существенные ограничения: метод ближайших соседей приводит к некорректным результатам, метод вспомогательной системы применим только в случае однонаправленного характера связи, а расчет показателей Ляпунова дает удовле-

Письма в ЖТФ, 2018, том 44, вып. 19 

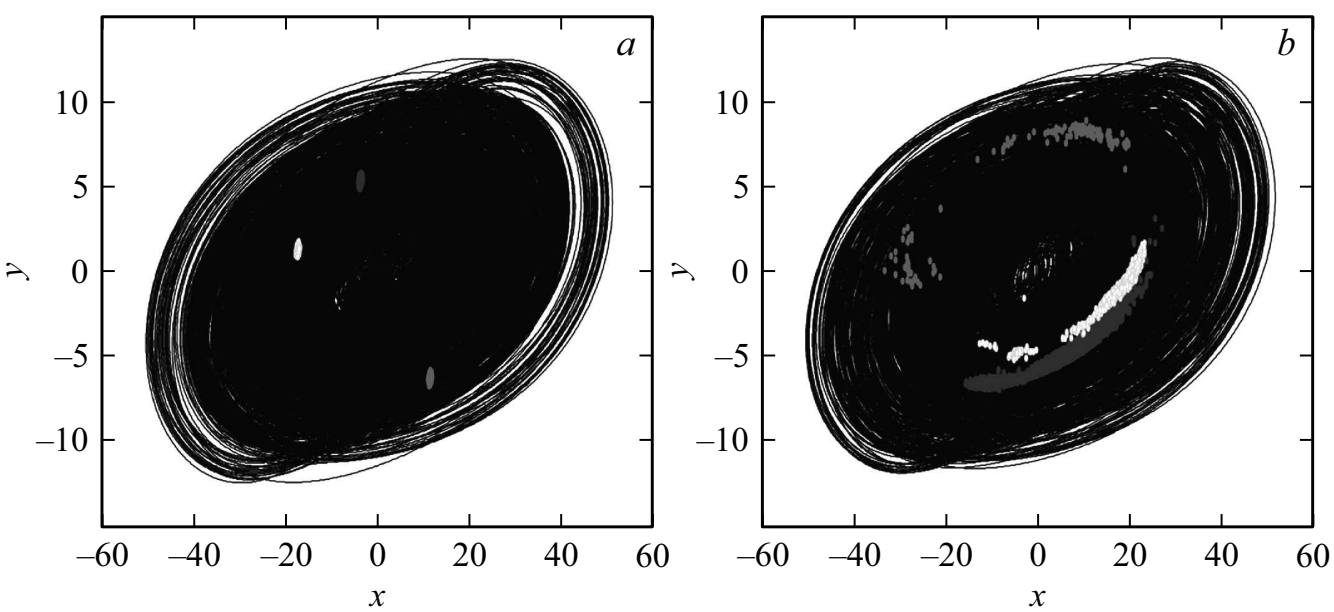

Рис. 2. Фазовые портреты первой $(a)$ и второй $(b)$ взаимодействуюших модифишированных систем Лоренца при значении параметра связи $\varepsilon=250$. На фазовом портрете первой системы (часть $a$ ) выбраны три случайные опорные точки и найдены их ближайшие соседи (показаны оттенками серого). Образы этих ближайших соседей в фазовом пространстве второй системы показаны теми же оттенками серого на части $b$. 

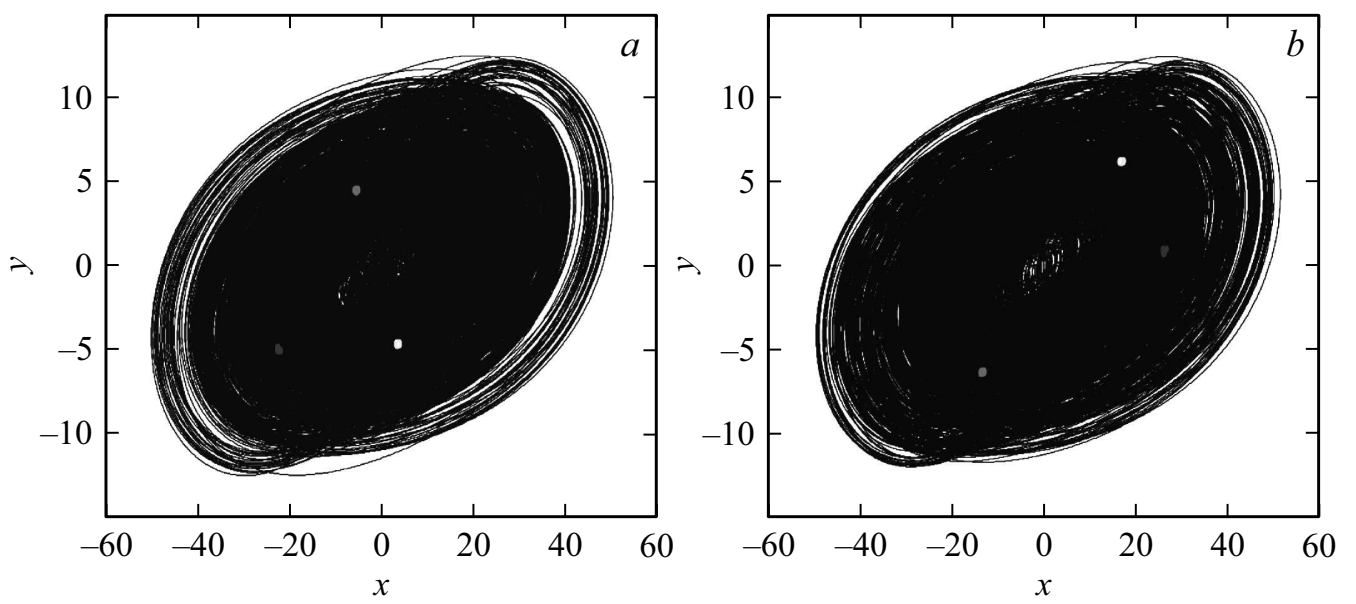

Рис. 3. Результаты применения метода фазовых трубок к модифицированным системам Лоренца. Фазовые портреть первой $(a)$ и второй $(b)$ взаимодействующих модифицированных систем Лоренца получены при значении параметра связи $\varepsilon=250$. На фазовом портрете первой системы (часть $a$ ) выбраны три случайные опорные точки и найдены их ближайшие соседи, прошедшие через фазовую трубку длиной $\tau=0.03$ (показаны оттенками серого). Образы этих „ближайших“ соседей в фазовом пространстве второй системы показаны теми же оттенками серого на части $b$. 
творительные результаты только в том случае, когда известен явный вид оператора эволюции. Важно подчеркнуть, что принципиальным достоинством метода фазовых трубок по сравнению с методом расчета спектра показателей Ляпунова является возможность его применения к экспериментальным временны́м рядам, в то время как расчет младших показателей Ляпунова по временно́му ряду представляется затруднительным. В связи с этим можно ожидать, что предложенный подход сможет найти применение в различных областях науки и техники, включая информационно-телекоммуникационные системы и нейрофизиологические задачи, в основе которых лежат анализ и обработка больших объемов данных.

Работа выполнена при поддержке Совета по грантам Президента РФ для государственной поддержки молодых российских ученых кандидатов наук (проект № МК-531.2018.2).

\section{Список литературы}

[1] Rulkov N.F., Sushchik M.M., Tsimring L.S., Abarbanel H.D.I. // Phys. Rev. E. 1995. V. 51. N 2. P. $980-994$.

[2] Стародубов А.В., Короновский А.А., Храмов А.Е., Жарков Ю.Д., Дмитриев Б.С. // Письма в ЖТФ. 2007. Т. 33. В. 14. С. 58-65.

[3] Koronovskii A.A., Moskalenko O.I., Hramov A.E. // Phys. Rev. E. 2011. V. 84. N 3. P. 037201.

[4] Moskalenko O.I., Koronovskii A.A., Hramov A.E., Boccaletti S. // Phys. Rev. E. 2012. V. 86. N 3. P. 036216.

[5] Короновский А.А., Москаленко О.И., Храмов А.Е. // Письма в ЖТФ. 2012. T. 38. B. 20. C. 21-29.

[6] Terry J.R., VanWiggeren G.D. // Chaos, Solitons and Fractals. 2001. V. 12. N 1. P. $145-152$.

[7] Короновский А.А., Москаленко О.И., Храмов А.Е. // УФН. 2009. Т. 179. № 12. C. 1281-1310.

[8] Prokhorov M.D., Ponomarenko V.I., Kulminskiy D.D., Koronovskii A.A., Moskalenko O.I., Hramov A.E. // Nonlinear Dynamics. 2017. V. 87. N 3. P. 2039-2050.

[9] Abarbanel H.D.I., Rulkov N.F., Sushchik M.M. // Phys. Rev. E. 1996. V. 53. N 5. P. 4528-4535.

[10] Pyragas K. // Phys. Rev. E. 1997. V. 56. N 5. P. 5183-5188.

[11] Moskalenko O.I., Koronovskii A.A., Hramov A.E. // Phys. Rev. E. 2013. V. 87. N 6. P. 064901. 
[12] Uchida A., McAllister R., Meucci R., Roy R. // Phys. Rev. Lett. 2003. V. 91. N 17. P. 174101.

[13] Короновский А.А., Москаленко О.И., Максименко В.А., Храмов А.Е. // Письма в ЖТФ. 2011. Т. 37. В. 13. С. 40-47.

[14] Wolf A., Swift J., Swinney H. L., Vastano J. // Physica D. 1985. V. 16. N 3. P. 285-317.

[15] Лоренц Э. Странные аттракторы / Под ред. Я.Г. Синая, Л.П. Шильникова. М.: Мир, 1981. С. 88-116.

[16] Chua L.O., Komuro M., Matsumoto T. // IEEE Trans. Circuits Syst. 1986. V. 33. N 11. P. 1073-1118.

[17] Анищенко В.С., Нейман А.Б. // Письма в ЖТФ. 1991. Т. 17. В. 14. С. 43-47.

[18] Zengqiang C.A., Yong Y., Guoyuan Q., Zhuzhi Y. // Phys. Lett. A. 2007. V. 360. N 6. P. 696-701. 